\title{
The Research and Implementation of PLC in Tobacco Blending Control System
}

\author{
Xiaoyu LIU ${ }^{1, a}$, Xianchong GUO ${ }^{1, b}$, Yueqiu YOU ${ }^{1, c}$ \\ ${ }^{1}$ Zhangjiakou Vocational and Technical College,China \\ a594265400@qq.com, b35541129@qq.com, ${ }^{\mathrm{c}} 709734051 @ q q . c o m$
}

\begin{abstract}
Keywords: tobacco production; tobacco blending; PID control; PLC; tobacco blending control system

Abstract: This paper expounds the selection of hardware, parameter Settings and software design in the PLC control system of the tobacco blending control system. It introduces the equipments composition, the control process, the method of flow control and the PID regulation of the tobacco blending technology during the tobacco processing in cigarette factories.
\end{abstract}

\section{Introduction}

Generally, cigarette tobaccos are composed of five tobaccos, they are leaf tobacco, peduncle tobacco, expansion tobacco, slice tobacco and recycling tobacco, and each cigarette products are prescribed the mixed proportion of the five components when design. The blending tobacco system are consist of PLC, sub -station, frequency changer, moisture meter and five nuclear scales that are leaf scale, peduncle scale, expansion scale, slice scales and recycling scales respectively, the leaf scale is the main scale called LCS-LL, the other four are auxiliary scales called proportional scale. he four auxiliary scales determine whether need to add and how much to add according to the technical index of different cigarette brands respectively and the flow of main scale. The accuracy of blending affect the different process indicators of the finished tobacco and the smoking taste of the finished cigarette directly, it plays a vital role on the inner quality of cigarette.

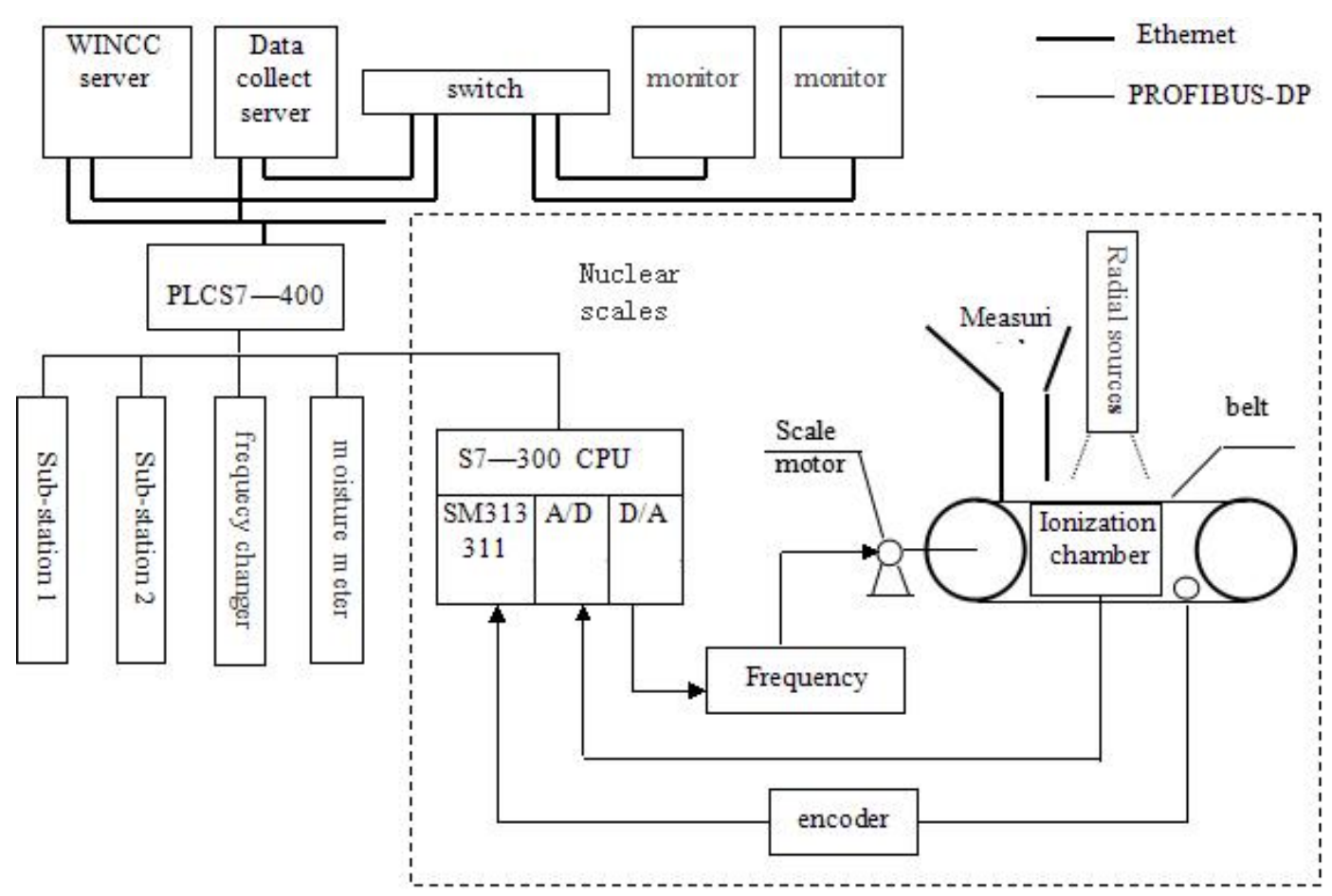

Fig.1 Configuration of the equipment in the blending tobacco process section 


\section{Blending tobacco process}

\section{1 Configuration of the blending tobacco process section control system}

the blending tobacco process section control system consists of central monitoring computer, programmable controller PLC(the main station), sub-station, frequency changer, moisture meter, nuclear scales and some related network[1]. It is shown in Fig.1.

\section{2 Control process in the blending tobacco section.}

Firstly, the System is initialized, the initial state is set, reset the memory block etc.. the upper PC of center control will set the blending ratio of each scale, and pass them to the PLC of on the network before production. PLC measures and controls in real time when the nuclear scales weigh the tobacco and detect, display and alarm faults, output signal to the change inverter at the same time , in order to adjust the motor speed and control the blending precision real-time. The nuclear scales mainly receive the information sented from the PLC in the process section, such as start, stop, and blending ratio etc.. and sent the cumulative weight, instantaneous flow and other status data back to the PLC through field bus. The nuclear scales receive the output signal of PLC in this process section to adjust the motor speed. if there is deviation between the instantaneous flow and the setting flow the controller compare the deviation between the set value and actual value according to the requirements of system control and change output signal through the PID adjustmen[2]t, control the speed adjustment of belt scale by frequency converter, realize the flow tracking, so as to achieve the control of the real-time blending accuracy, blending proportion can be modified according to the technics requirement.

\section{3 PID regulation of nuclear scale's flow}

The five nuclear scales in the blending tobacco process section provide material through the measuring tube. The system enter the external control mode after power on, and all the nuclear belt scales is in the stopped state. The five nuclear scales begin run when receive the start signal on the whole line, but the instantaneous flow rate is zero as there is no raw material on the main leaf tobacco scale, and the setting flow of four others is 0 , so the belt motor frequency of the four auxiliary scales' is 0 . Under normal circumstances, in order to ensure the blending of various auxiliary scales, material will be placed on the auxiliary weighting-scales before the material on the leaf tobacco main scale come. peduncle tobacco, expansion tobacco, slice tobacco and recycling tobacco are placed at the exit of the belt in advance waiting for the material on the leaf tobacco scale coming. When the material on the main leaf scale is incoming, the other 4 nuclear scales will set flow based on a certain proportion of the instantaneous flow on the main scale, adjust the instantaneous speed of belt scale so as to adjust the instantaneous flow of nuclear scale by controlling the motor operating frequency of the belt scale and track the set flow ccording to the delay time. The flow is the amount of material carried through the belt per unit time. The nuclear belt scale will calculate the instantaneous flow , the central PC will give the setting percentage and the setting flow will be calculated on local machine[3]. because there may be deviation in real-time operation in the results the flow is controlled by the PID regulation which is most widely used in industrial control. It calculates the control quantity by using the proportion, integral and differential according to the flow deviation. The PID controller has become one of the main technology of industrial control for its simple structure, good stability, reliable work and convenient adjustment. PID close-loop control of nuclear scale flow is shown Fig.2.

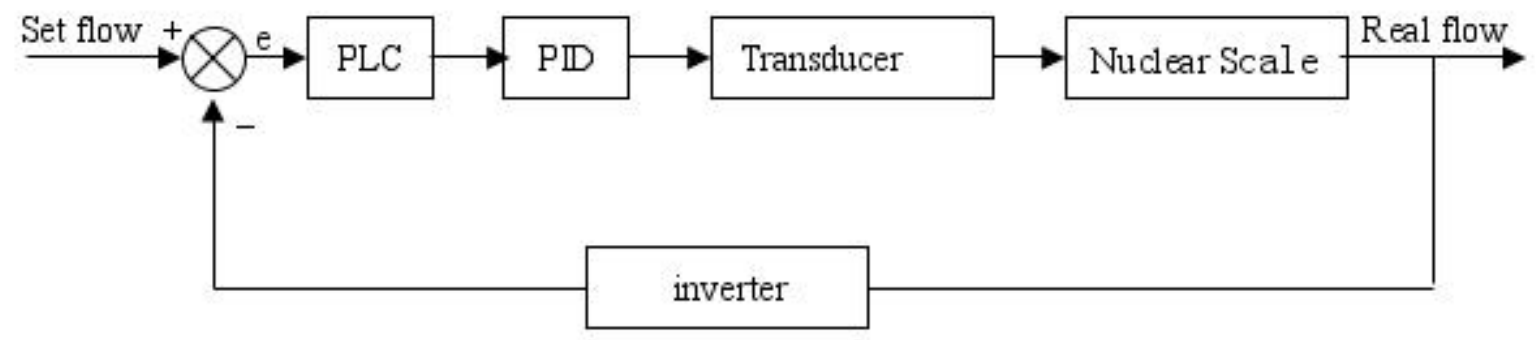

Fig. 2 PID close-loop control of nuclear scale flow 


\section{Configuration of the hardware in PLC}

There are the programmable S7-400 in the main control room, computer inside the control room and five nuclear belt scales which has a programmable controller S7-300, a motor of scale, a speed sensor, a radiation source, an ionization chamber and a frequency converter. They are the basic unit in the whole control system of detection and execution. the system needs to process the digital data which is the st art, stop of motor, the chain signal and failure information comes from the equipments on production line, the analogue data which are weight, flow and inverter controlled signal and the high-pulse signal which is the speed signal real-time.

\section{Selection of programmable controller}

\section{1 SIMATIC s7-400}

Given the number of the input / output, process requirements, software capacity, running speed Etc., SIMATIC S7-400 is used as the programmable controller in main controller. It is built to a modular design, with wide properties, flexible combination with different templates and convenient extension. A system may include[4]:

- $\quad$ Power template (PS): connect the SIMATIC S7-400 to 120/230VAC or 24DC power supply.

- $\quad$ The central processing unit (CPU): with built-in interface of PROFIBUS-DP , used for a variety of performance, can include multiple CPU to enhance its performance.

- $\quad$ Signal template (SM): the digital input and output (DI/DO) and analog input and output (AI/AO).

- $\quad$ Communication processor (CP): used for bus and point-to-point connection.

- $\quad$ Function template (FM): specially used for counting, positioning, cam control task.

\section{2 SIMATIC S7-300}

SIMATIC S7-300 is used as nuclear scale programmable controller. It is a medium or small PLC system with modular design, it can meet medium requirements application, easy to distribute and master. The system may include:

- $\quad$ The central processing unit (CPU): CPU has integrated input / output points, PROFIBUS-DP.

- $\quad$ Signal module (SM): used for input / output of digital and analog .

- Communication processor (CP): used to connect to the network and the point-to-point connection.

- $\quad$ The function module (FM): used for high-speed counting, positioning operation (open or closed loop control )

- $\quad$ Load power module (PS): used for the connection of SIMATIC S7-300 to 120/230V AC.

- Interface module (IM): used for the connection of the main frame (CR) to expansion (ER) when there is multiple configurations.

\section{3 The choice of converter}

At present, the Series VLT 2800 is one of the most compact multifunctional inverter on the market. It is more suitable for installed side by side to save space greatly, and it can match the motor coil, RF interference filter, LC+1B filter components. It has optional communication interface module which usually used in industrial field bus includes the DeviceNet, ModBus RTU, etasys N2, ROF IBus DP and others and easy to expand the system's functions. Three-phase AC motor of $0.75 \mathrm{~kW}$ is used by the 5 belt scales, the inverter's power out is $0.37 \sim 1.5 \mathrm{~kW}$. In the system.

\section{Software design of PLC control system}

The programming of S7- 400, S7-300 series programmable controller is based on SIMATIC MANGER STEP7 integrated development environment which is a program development environment running on the WINDOWS. ladder diagram and sentence table and other forms of programming can be easily used by the STEP7, and then the compiled software is loaded down in PLC to run. Also the running state can be monitored online in order to debug the software faclitely. 
STEP7 divide the user program into system block and user. block. The user block includes: organization block (OB), function block (F B), function (FC), data block(DB). The OB is called by the system block to complete different functions, the FB is called by the $\mathrm{OB}$, the user data is stored in the DB to supported the save function when power off.

\section{1 Organization block (OB)}

- $\quad$ S7-400 series: OB1, OB32, OB35, OB100, OB8187, OB101, OB102, OB121/122

- $\quad$ S7-300 series: OB1, OB35, OB82, OB85-87, OB100, OB121/122

\section{2 Function(FC)}

It include sequential control, special control, motor parameter control, process control, and communication with HMI , function subprogram Communication with Ethernet.

\section{3 The function block (FB)}

It including Siemens system function blocks, the blocks developed independently to realize scale measurement and mixing function: measurement block, automatic control block, PID peeling block[5], calibration block, chain logic control block, network communication processing block, alarm function block.

\section{4 Data block (DB)}

It including special function control block, motor parameter block, process control block, data block communicating with HMI, Ethernet communication data blocks, DP network communication, DP network diagnosis.

\section{Conclusion}

The actual operation of the mixing tobacco system indicates that the designation and installation of the system is reasonable, running stable and reliable. the traditional relay logic is replaced by the programmable logic control PLC that not only improves the reliability of the whole system, but also improve the accuracy of measurement and control of nuclear belt scales because of its powerful function, convenient and flexible, easily to maintained Etc., and it supply the conditions for the whole processing tobacco workshop to network.

\section{References}

[1] Dr Jie Zhao, Dunstan Thomas Conculting, Robust Object Oriented System Analysis, http://consulting.dthomas.co.uk

[2] Elmasi R., Navathe S.B. Fundamentals of Database Systems, The Benjamin/Cummings Publishing Company, Inc.(1989)

[3] Khaled Kamel, Eman Kamel, Programmable Logic Controllers Industrial Control, third ed., Mechanical Industry Press (2015)

[4] Changchu LIAO, The Application Technology of PLC S7-300/400, third ed., In Chinese , Mechanical Industry Press (2012)

[5] BECKHOFF, TwinCAT PLC Control(2005), Information on http://wenku.baidu.com/ 